\title{
Implementing a novel protocol for withdrawal of CPAP support in COVID-19 patients: a case series
}

\author{
Authors: Natalie Webber, ${ }^{\mathrm{A}}$ Malcolm Avari, ${ }^{\mathrm{B}}$ Georgina Harridge, ${ }^{\mathrm{C}}$ ]ane Neerkin, ${ }^{\mathrm{D}}$ Emily Collis $^{\mathrm{D}}$ and Ronan Astin ${ }^{\mathrm{E}}$
}

\section{Background}

No published protocol to guide the withdrawal of continuous positive airway pressure (CPAP) for patients with COVID-19 exists.

\section{Case series}

Description of the introduction of a novel protocol, developed by consensus to guide the withdrawal of CPAP for patients diagnosed as dying with COVID-19 in an acute hospital.

\section{Outcome}

19 patients died on the high-dependency respiratory unit following treatment with CPAP. $89 \%$ died with CPAP withdrawn. The dying trajectory was difficult to predict. Symptoms were managed promptly and effectively with a combination of opioids, benzodiazepines and close medical supervision. No concerns were raised by families regarding the decision making or withdrawal process.

\section{Discussion}

The use of the protocol ensures a comfortable and dignified death and supports the delivery of individualised care at the end of life. Future research on this topic should focus on qualitative outcomes and consider the applicability of this protocol in other patient groups.

KEYWORDS: Case series, COVID-19, continuous positive airway pressure, withdrawal, palliative care

DOI: $10.7861 /$ clinmed.2020-1086

\section{Background}

Continuous positive airway pressure (CPAP) support has a proposed role in the management of respiratory failure caused

Authors: Aspecialist registrar in palliative medicine, University College London Hospitals NHS Foundation Trust, London, UK; ${ }^{B}$ specialist registrar in respiratory medicine, University College London Hospitals NHS Foundation Trust, London, UK; ' foundation year doctor, University College London Hospitals NHS Foundation Trust,


London Hospitals NHS Foundation Trust, London, UK; ${ }^{\text {Econsultant }}$ in respiratory medicine, University College London Hospitals NHS Foundation Trust, London, UK by coronavirus disease 2019 (COVID-19) as a strategy to avoid intubation, as a ceiling of treatment and perhaps less commonly to facilitate extubation. ${ }^{1}$ Despite an overall mortality risk from COVID-19 that is estimated to be between $3-5 \%,{ }^{2}$ with intensive care mortality rates close to $40 \%$ by the end of May $2020,{ }^{3}$ published guidance regarding the use of CPAP fails to address how to withdraw this support in patients for whom invasive ventilation is not appropriate and are approaching the end of life. Such guidance is necessary in order to maximise comfort and dignity?

On review of the literature, the available guidance regarding the withdrawal of non-invasive respiratory support largely relates to patients with motor neurone disease. This is a distinct patient population, but there are some parallels with CPAP withdrawal in the setting of COVID-19; dyspnoea and anxiety can be expected and as such these symptoms should be anticipated and managed proactively with a combination of opioids and benzodiazepines. ${ }^{4}$

During a pandemic such as COVID-19, health systems are stretched, clinicians are redeployed and there is the potential for rapid patient deterioration. Standardised protocols can help empower non-specialists to provide high-quality end-of-life care to ensure that patients die comfortably and with dignity. ${ }^{5}$ To achieve appropriate symptom control when withdrawing CPAP, it is paramount that any proposed framework allows clinicians to be responsive to the individualised needs of the patient. ${ }^{4}$ This case series describes the experience of implementing a novel protocol developed for the withdrawal of CPAP support in COVID-19 patients in one London-based acute hospital trust.

\section{Case series}

On the high-dependency respiratory unit (HDRU) there were a total of 70 patients who required CPAP as part of the first wave of COVID-19 (end March to end June 2020). Of these patients, 19 received end-of-life care on the unit. These patients were deemed not for further escalation to intensive care nor for resuscitation for a variety of reasons including patient choice, comorbidities, frailty, poor physiological reserve and poor overall prognosis. The comorbidities noted were wide-ranging but primarily cardiovascular, with hypertension being the most common, affecting $68 \%$ of patients in this cohort. Two patients who had CPAP withdrawn had no known comorbidities. The mean duration of CPAP therapy was 8.2 days, with $37 \%$ receiving CPAP for more than 10 days. The palliative care team attended daily board rounds on the unit to assist with symptom control, advance care planning and end-of-life care. The vast majority of the CPAP withdrawals 
Table 1. Demographics and clinical characteristics of patients who had CPAP withdrawn. $\mathbf{N}=19$

\begin{tabular}{ll} 
Age, mean (range) & $75(60-84)$ years \\
Length of stay, mean (range) & $12.1(5-27)$ days \\
Clinical Frailty Scale score, mean (range) & $4(1-7)$ \\
Duration of CPAP, mean (range) & $8.2(3-16)$ days \\
Maximum CPAP PEEP, mean (range) & $13(10-15) \mathrm{CmH}_{2} \mathrm{O}$ \\
Maximum $\mathrm{FiO}_{2}$, mean (range) & $90 \%(60-100 \%)$ \\
Ethnicity, \% BAME & $84 \%$ \\
CPAP withdrawal planned & $84 \%$ \\
$\begin{array}{l}\text { Daytime (9am-5pm) CPAP withdrawal } \\
\text { Comorbidities }\end{array}$ & $84 \%$ \\
$\quad$ Endocrine & $26 \%$ \\
Cardiovascular & $74 \%$ \\
Respiratory & $26 \%$ \\
Neurological & $32 \%$ \\
Haematological & $32 \%$ \\
Other & $89 \%$ \\
\hline BAME = Black, Asian or minority ethnicity; CPAP = continuous positive airway \\
pressure; FiO ${ }_{2}=$ fraction of inspired oxygen; PEEP = positive end expiratory \\
pressure
\end{tabular}

were planned, from a few hours to one day in advance. This meant that in most cases the patient and/or their family were aware of the plan, allowing final visits or phone/video calls to take place. It also allowed for face-to-face palliative care input in $84 \%$ of cases and for appropriate anticipatory medications to be administered in advance to maximise patient comfort. The two cases where palliative care was not involved at all were due to sudden and unexpected deteriorations out of hours. Table 1 demonstrates a summary of the key characteristics of this cohort of patients. Since this case series used anonymised data from clinical routine, no application for an ethics approval was required.

Prior to the emergence of COVID-19, no protocol for CPAP withdrawal existed within our Trust. The option to continue with no protocol was deemed not an appropriate course of action. It was recognised that a protocol was needed to support non-specialists to deliver quality care at the end of life.

The protocol for CPAP withdrawal (Fig 1) was developed by a small group of palliative medicine, intensive care and respiratory consultant physicians with input from senior nursing staff. The protocol was adapted following feedback and ratified by the local COVID-19 Steering Group, End-of-Life Care Board and clinical ethics advisory groups. A key consideration was the clinical diagnosis of dying as the first step of the protocol. The doses of morphine and midazolam suggested are higher than standard pro re nata (prn, as needed) doses prescribed routinely in end-of-life care, consistent with both evidence ${ }^{6}$ and clinical experience that COVID-19 patients with respiratory distress require higher than standard starting doses to effectively alleviate symptoms. The protocol is sufficiently detailed to guide relatively inexperienced clinicians through the steps (for example, stipulating that the CPAP machine is switched off before removing the mask) but incorporates flexibility to allow individualisation of care. Supporting family communication, involvement and support within the context of restricted face-to-face visiting, was paramount.
Recognise that patient is in last hours to short days of life

$$
\downarrow
$$

Refer to specialist palliative care (Bleep XXXX)

$$
\downarrow
$$

Discuss with family by telephone and commence individualised care plan for care in last days of life Check religious/spiritual needs with family

$$
\downarrow
$$

Invite one family member who is not self-isolating to visit patient prior to CPAP withdrawal (with PPE); alternatively offer telephone/video call

\begin{tabular}{c}
$\downarrow$ \\
\hline $\begin{array}{c}\text { Cease monitoring }\left(\mathrm{BP} / \mathrm{O}_{2} \text { saturation/cardiac) }\right. \\
\text { and complete symptom assessment }\end{array}$ \\
\hline
\end{tabular}

Give stat dose of morphine $5 \mathrm{mg}$ SC/IV + midazolam $5 \mathrm{mg} \mathrm{SC/IV}$
+ /- levomepromazine $6.25 \mathrm{mg} \mathrm{SC}$
$\downarrow$

Commence CSCI midazolam $20 \mathrm{mg}$ and morphine $20 \mathrm{mg}$ over 24 hrs (if already on CSCI titrate to symptoms) $\downarrow$

Consider weaning down oxygen via CPAP to $21 \%$ (if patient appears comfortable) $\downarrow$

Consider weaning down CPAP pressure support. (if patient appears comfortable)

\section{$\downarrow$}

Reassess effects of sedation ( $<15 \mathrm{~min}$ post SC injection) and whether patient comfortable to remove mask (eg if patient has tolerated weaning of oxygen and pressure support).

Give repeat prn doses of morphine 5-10 mg SC/IV and midazolam 5-10 mg SC/IV if needed

\section{$\downarrow$}

Turn off CPAP machine and then remove CPAP mask

$$
\downarrow
$$

Consider giving oxygen $10 \mathrm{~L} / \mathrm{min}$ via non-rebreathe mask, reducing to $5 \mathrm{~min} / \mathrm{L}$ after $15 \mathrm{~min}$, reducing further to $2 \mathrm{~L} / \mathrm{min}$ after further $15 \mathrm{~min}$ and then ceasing (as tolerated)

\section{$\downarrow$}

Reassess symptom control and give repeat prn doses morphine 5-10 mg SC/IV + midazolam 5-10 mg SC/IV + /- levomepromazine $12.5 \mathrm{mg}$ SC if needed

Fig 1. Protocol for CPAP withdrawal for COVID-19 patients at the end of life. $\mathrm{BP}=$ blood pressure; $\mathrm{CPAP}=$ continuous positive airway pressure; $\mathrm{CSCI}=$ continuous subcutaneous infusion; $\mathrm{IV}=$ intravenous; $\mathrm{PPE}=$ personal protective equipment; $\mathrm{prn}=$ pro re nata (as needed); $\mathrm{SC}=$ subcutaneous.

\section{Outcome}

The palliative care team led the CPAP withdrawal in 10 of the 19 patients. The respiratory team led a further six CPAP withdrawals (the majority of these out of hours). Additionally, one patient died 
very suddenly while on CPAP during a palliative care review. One patient, in keeping with his family's wishes, continued CPAP for comfort during end-of-life care and one patient removed the CPAP himself and declined to have it replaced. Some patients had prior referrals to the palliative care team for symptom management of their breathlessness or anxiety induced by the CPAP therapy.

Where possible, a family member/friend was offered the opportunity to have a face-to-face discussion with the multidisciplinary team and then to visit the patient. In seven cases family were present for the withdrawal, and in one further case they opted to participate by video call during the process.

A formal individualised end-of-life care plan was created for 11 of the 19 patients, including referral to hospital chaplaincy where appropriate. The patients were all too unstable to move out of the hospital, even if their preference had been to die at home. Monitoring was stopped and symptom control observations were commenced. All the patients were tachypnoeic and were given prn doses of midazolam and either morphine or oxycodone. Five patients who were extremely agitated and distressed were given the medication by slow intravenous injection and the remaining 14 patients were given the medication subcutaneously. Levomepromazine was added to prevent opioid-induced nausea and vomiting in patients who were opioid-naïve.

If the patient was not already on a continuous subcutaneous infusion (CSCI) of opioids and benzodiazepines to manage their symptoms, this was started with midazolam and morphine or oxycodone. 17 out of 19 patients were prescribed a CSCI. The oxygen was weaned down and then the pressure support. When the patient was judged to be comfortable with acceptable work of breathing, the CPAP mask was removed. Additional prn medication was available in case the patient became agitated or distressed at any point in the process.

On withdrawal of CPAP, patients tended to follow one of two dying trajectories: either a very rapid decline leading to death within 10-15 minutes or a more prolonged deterioration over the course of a few hours. It was difficult to predict which trajectory patients would follow, but it was observed that those with higher levels of agitation and confusion prior to withdrawal requiring intravenous symptom control rather than subcutaneous tended to deteriorate faster. In our cohort, 4 out of 19 patients experienced a very rapid decline. The longest times from withdrawal to death were in patients who spontaneously deteriorated with reduced conscious level prior to the withdrawal, with one patient surviving 25 hours and another 4 days.

In all cases, symptoms were managed promptly and effectively and patients were comfortable at the time of their death. In the subsequent bereavement follow up, no concerns were raised regarding the decisions around, or process of, CPAP withdrawal (calls documented to 13 of the 19 bereaved families). However, in two cases there were concerns raised around earlier treatment escalation planning conversations, regarding decisions around invasive ventilation and resuscitation status.

\section{Discussion}

This is the first case series to describe the experience of CPAP withdrawal in a COVID-19 patient cohort using a novel protocol specifically developed for this purpose. Recognition of patient deterioration despite maximal medical therapy should trigger a referral to specialist palliative care to support the development of an individualised plan for care at the end of life. A multidisciplinary team approach, ascertaining patient wishes and ensuring family involvement are of paramount importance in the delivery of holistic care. The protocol for CPAP withdrawal helps ensure a comfortable and dignified death for all patients, even when the clinicians involved are inexperienced in the process. The clinical trajectory following withdrawal of CPAP is difficult to predict, necessitating close medical supervision to ensure the timely administration of intravenous midazolam for patients where there is insufficient time for effective symptom control using subcutaneous medication.

This case series supports the use of a CPAP withdrawal protocol for future COVID-19 surges. The protocol could be adapted for other conditions where the withdrawal of non-invasive ventilation at the end of life may be appropriate (for example, in patients with COPD or motor neurone disease). Future research on this topic should consider qualitative outcomes and the wider applicability of this protocol.

\section{Summary}

\section{What is known?}

No previous literature examines the use of a protocol to guide the withdrawal of CPAP in patients with COVID-19. Existing literature largely relates to patients with motor neurone disease.

\section{What this paper adds}

This case series demonstrates that the use of a protocol to guide the withdrawal of CPAP in patients with COVID-19 ensures a comfortable and dignified death without compromising the delivery of individualised care at the end of life.

\section{Implications for practice, theory or policy}

This case series supports the use of a novel protocol to guide withdrawal of CPAP for patients with COVID-19 who have been diagnosed as dying. Future research should consider applicability for other patient groups.

\section{References}

1 National Health Service (NHS). Guidance for the role and use of non-invasive respiratory support in adult patients with coronavirus (confirmed or suspected). NHS, 2020. https://anaesthetists.org/ Portals/O/PDFs/COVID-19/CLEARED_Specialty-guide_-NIVrespiratory-support-and-coronavirus-v2-26-March-003.pdf [Accessed 12 June 2020].

2 Baud D, Qi X, Nielsen-Saines K et al. Real estimates of mortality following COVID-19 infection. Lancet Infect Dis 2020;20:773.

3 Armstrong RA, Kane AD, Cook TM. Outcomes from intensive care in patients with COVID-19: a systematic review and meta-analysis of observational studies. Anaesthesia 2020;75:1340-9.

4 Association for Palliative Medicine. Withdrawal of assisted ventilation at the request of a patient with motor neurone disease. APM, 2015. www.apmonline.org/wp-content/uploads/2016/03/ Guidance-with-logos-updated-210316.pdf [Accessed 12 June 2020].

5 Downar ], Seccareccia D. Palliating a pandemic: 'all patients must be cared for'. J Pain Symptom Manage 2010;39:291-5.

6 Association for Palliative Medicine. COVID-19 and palliative, end of life and bereavement care in secondary care (Version 4). APM, 2020. www.apmonline.org/wp-content/uploads/2020/04/ COVID-19-and-Palliative-End-of-Life-and-Bereavement-Care-20April-2020-2.pdf [Accessed 24 November 2020].

Address for correspondence: Dr Natalie Webber, Palliative Medicine, 2nd Floor Wing B, Maple House, 149 Tottenham Court Road, London, W1T 7NF, UK.

Email: nataliewebber@nhs.net 